\title{
Integrative Medicine at Jefferson: Leading the Way with New Center and Cutting-Edge Technology
}

\section{Jane Hart, MD}

\begin{abstract}
The Myrna Brind Center of Integrative Medicine at Jefferson University Hospitals, Thomas Jefferson University, Philadelphia, Pennsylvania, has combined its expertise, experience, and advanced diagnostics to serve as a leader in the field of integrative medicine and provide state-of-the-art care for people desiring an integrative medicine
\end{abstract} approach to their health. Now, the recent awarding of a \$14 million grant by the Marcus Foundation, Inc., has allowed for the establishment of a

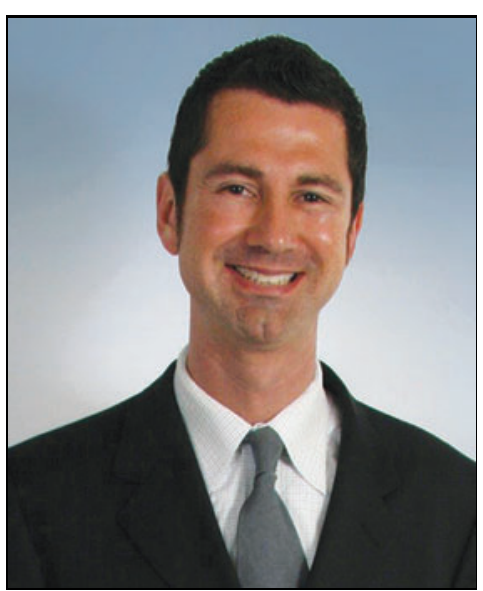

Daniel A. Monti, MD, MBA, Senior Vice President and Director of Jefferson's Myrna Brind Center of Integrative Medicine and Brind-Marcus Center of Integrative Medicine. new center, the Brind-Marcus Center of Integrative Medicine, in Villanova, Pennsylvania, which opened its doors in December 2015. The new Center has enabled expansion of both clinical and research endeavors as well as cutting-edge technology, including the addition of a unique positron emission tomography-magnetic resonance imaging (PET-MRI) scanner.

Daniel A. Monti, MD, MBA, senior vice president and the director of both centers, commented on the evolution of success of integrative medicine at Thomas Jefferson University:

There has been a real transformation over the past decade at Jefferson, where integrative medicine is now definitely part of the fabric of the enterprise and a highly featured and well-positioned department in the institution. Each integrative medicine center throughout the country may be very different in terms of clinical and research offerings. Our approach is to look through the lens of modern medicine within a holistic framework and serve as a consultation service for all. Now, through the generosity of the Marcus Foundation, we have expanded our current capabilities with a broader geographic reach to provide expertise in therapeutic options beyond conventional medicine, such as targeted nutrition, lifestyle change, and advanced diagnostic technology. We could not be more excited about this.

Kathleen Gallagher, executive vice president, chief operating officer, and chief corporate officer of Thomas Jefferson University commented: "Our integrative medicine initiative has been a very successful program, with excellent patient feedback, and now the demographics of having a Center in a suburban setting provides more access to individuals who are looking for this type of care." She added: "Our institution fully supports the presence and expansion of integrative medicine within our Centers, and we are pleased to be able to provide board-certified physicians and expert clinicians to approach our programs and expansion from an evidence-based and scientifically sound foundation."

\section{Expansion of Services and Cutting-Edge Technology}

The new 14,000-square-feet Brind-Marcus Center of Integrative Medicine in Villanova, PA, represents a major expansion of integrative health services at Jefferson, which builds upon the successful model created by their Philadelphia facility, The Myrna Brind Center of Integrative Medicine. The Brind-Marcus Center of Integrative Medicine will provide new as well as similar services as the main campus including: oneon-one consultations with integrative medicine experts; extensive integrative medicine programming; advanced diagnostics capabilities with the new PET-MRI scanner; and an infusion center. Dr. Monti commented: "We provide a very careful and thoughtful assessment with the patients we see, which is comprehensive and offers a lot of one-on-one physician time. However, in addition to a thorough head-to-toe assessment, we also spend considerable time on stress 


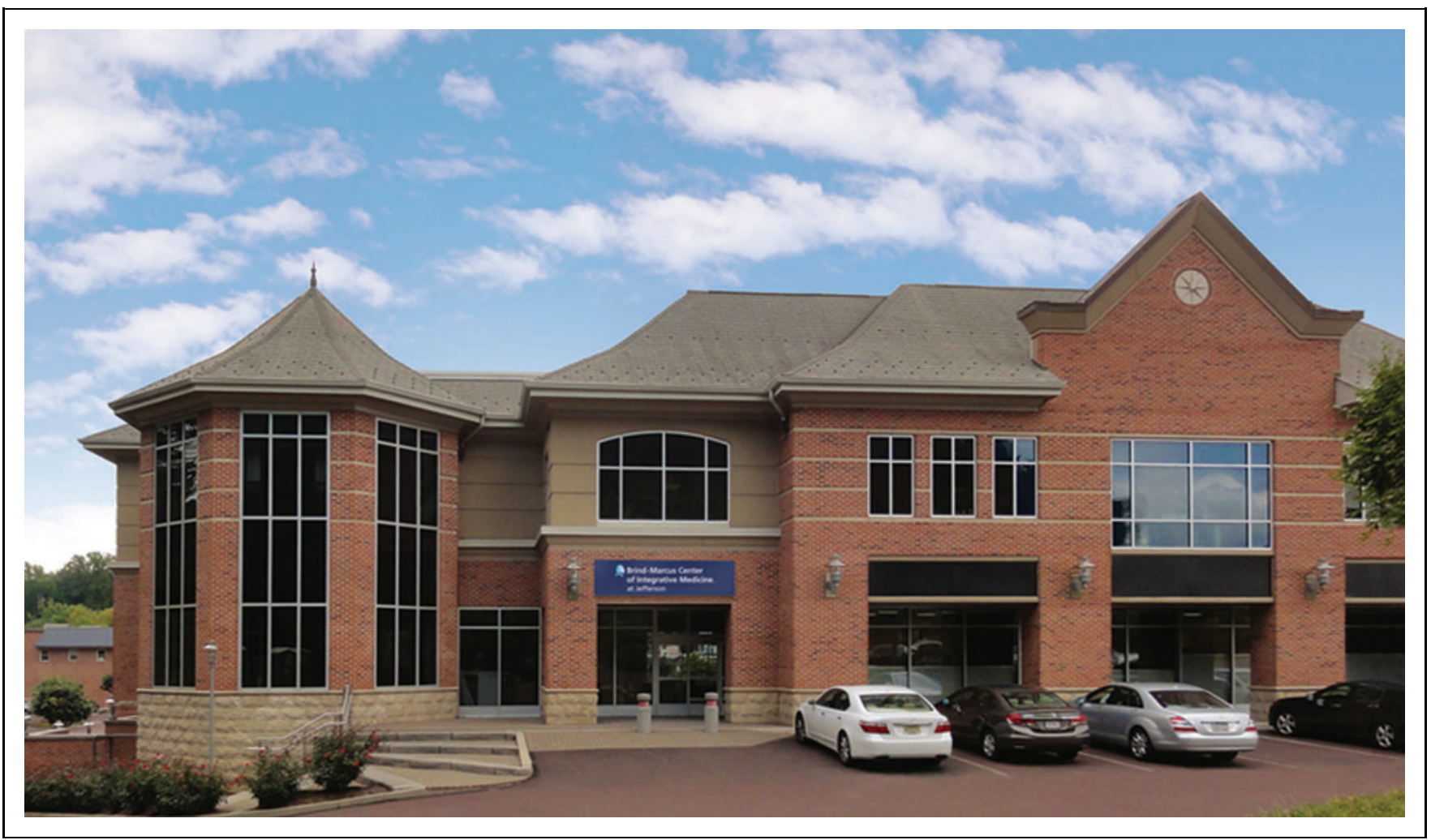

The Brind-Marcus Center of Integrative Medicine, in Villanova, Pennsylvania.

management, daily intake of nutrients, and other important lifestyle variables."

Featuring holistic medical and wellness services, the depth and breadth of program offerings at both the Center City Philadelphia and Villanova Centers is significant and includes: an integrative executive health program; integrative chronic illness management; integrative cardiology; a neurocognitive (Executive Brain Health) program; micronutrient infusion therapies; natural hormone therapies; acupuncture; a smoking cessation program; a wide range of mindfulness-based stress reduction (MBSR) programs; and others.

Sabrina Bazzan, JD, MBA, administrative and operations director at the Myrna Brind Center and at the Brind-Marcus Center of Integrative Medicine stated: "Our executive health program-Executive Great Life-is a key service that is offered at both locations and is one of the only executive health programs in the nation that is rooted in integrative medicine." Dr. Monti added: "We have an increasing number of companies signing on to have their executive team[s] receive yearly physicals through this program. This has been a stand-alone service that has done very well and has raised awareness about our Center and generated many referrals as well." The MBSR program, another flagship program, is one of the largest in the country, according to Dr. Monti, with the ability to certify trainers in MBSR. He states: "We have a great breadth of curriculum in mindfulness that has been a core part of the Center, from offering the 8-week standardized program, to certifying teachers, to specialized programs for mental health professionals, as well as workshops that we offer to medical students and other professionals."

\section{Advanced Diagnostic Technology}

One of the main features of the Brind-Marcus Center of Integrative Medicine is the new PET-MRI scanner which was also funded through the Marcus Foundation grant. Dr. Monti stated that the PET-MRI scanner at the Villanova Center is the first in the region, making it a distinguishing feature not only for integrative medicine at Thomas Jefferson University, but also for the entire institution. Dr. Monti explained:

The PET-MRI scanner allows us to do PET imaging with MRI instead of a [computed tomography] scan, and there are a lot of advantages to this. If we are looking at the brain, for example, we can obtain a picture of anatomy with the MRI and also get a sense of functional activity with the functional MRI. In addition, we can view yet another layer of functional activity with the PET scan, all in one hybrid, integrated machine without ever leaving the room.

Vijay M. Rao, MD, FACR, David C. Levin Professor and Chair, Department of Radiology, Sidney Kimmel Medical College at Thomas Jefferson University, commented: "Our new offering of the PET-MRI, the latest advancement in imaging technology, furthers our commitment to innovation and an integrative approach to patient care. The PET-MRI 


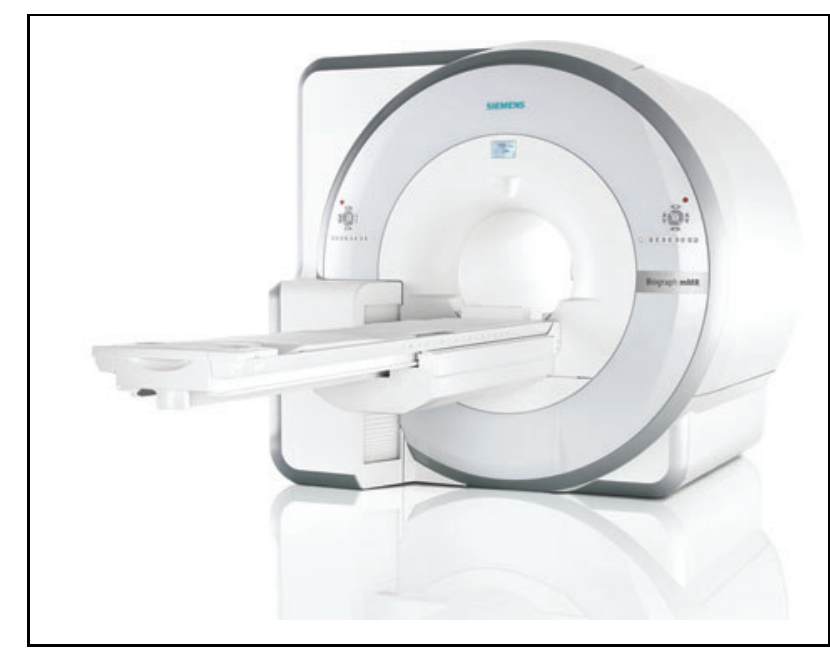

Positron emission tomography-magnetic resonance imaging scanner at the Brind-Marcus Center of Integrative Medicine.

allows a unique view of both anatomy and metabolic activity, providing a more complete investigation into our patients' conditions. This capability increases patient access to cuttingedge technology, and the information that a PET-MRI provides can also help close the gap between diagnosis and treatment."

Andrew B. Newberg, MD, professor of emergency medicine and radiology and director of research at both Centers commented on the new technology: "Clinically, the PETMRI scanner will help us to better evaluate patients in order to determine more effective therapeutic approaches for many of the conditions we treat." He added: "By using the PET-MRI scanner, we can obtain detailed diagnostic information to help guide therapy for conditions such as chronic fatigue, chronic pain, irritable bowel syndrome, cognitive impairment or cardiovascular risk. From a research perspective, the PET-
MRI will truly be a game changer in terms of helping to better understand the physiological effects of integrative therapies."

Dr. Newberg and his colleagues are planning an aggressive research program, which will use the PET-MRI scanner to help determine, for example, how proper diet and nutrition reduce inflammation; how specific supplements might improve cognitive function or help patients who have neurodegenerative disorders such as Parkinson's disease; and how practices, such as meditation, acupuncture, or other mindbody approaches, work to improve patients' health and well being. He said: "The ability to demonstrate the specific mechanisms by which these integrative approaches work will provide the necessary data to potentially shift medical attitudes about integrative medicine."

The Brind-Marcus Center of Integrative Health will also offer a neurocognitive assessment service (Executive Brain Health), where the technology of the PET-MRI is combined with neurocognitive testing, autonomic nervous system testing, and a neurological examination. Bazzan said, "I am very excited about our new Executive Brain Health Program, which will utilize this technology and provide the most comprehensive assessment of cognitive function and brain health available." In terms of how these services will specifically benefit patients, Dr. Monti commented: "One of our goals, for example, is that if a person is relatively healthy, but they want to maximize cognitive reserve, then we will take an integrative approach for the path forward, utilizing a range of options from diet and targeted nutrition to stress-reduction and brain-fitness programs."

For patients with postconcussive symptoms, the PET-MRI can help document areas of the brain that are abnormal in their function and then help tailor a treatment program-including diet, supplements, meditation, or other integrative approaches designed to help support brain function-according to Dr. Newberg. He commented: "The opportunities with the PET-

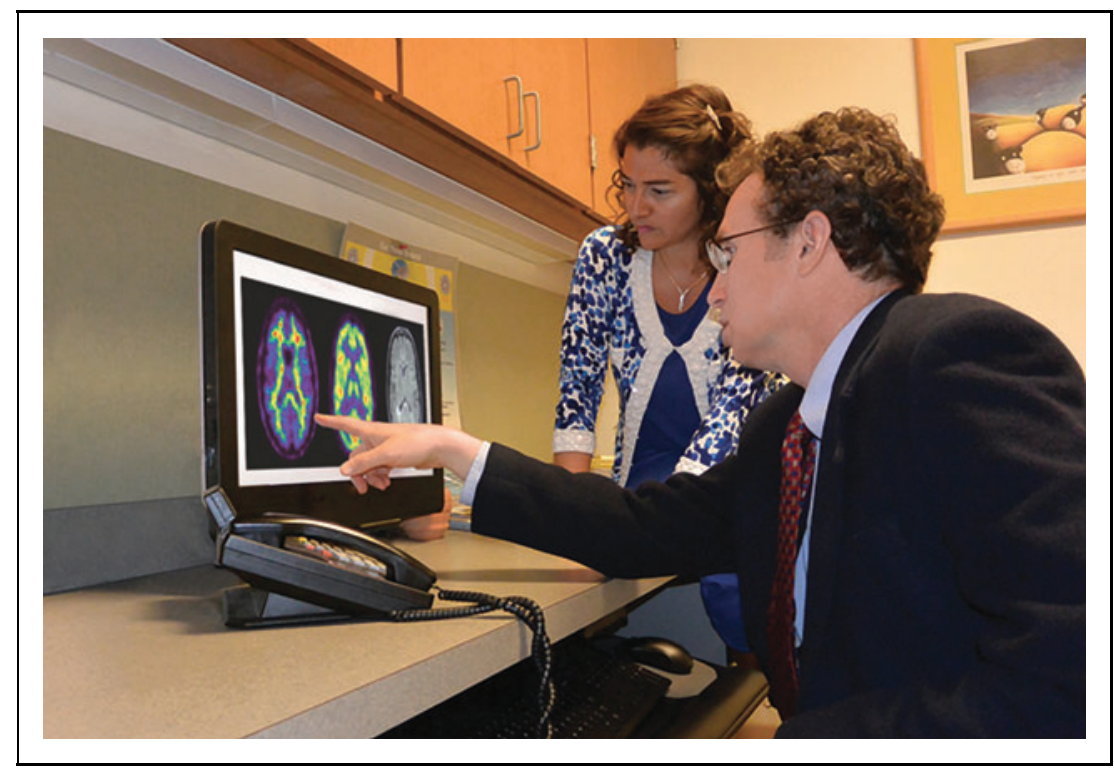

Andrew B. Newberg, MD, reviews a PET-MRI scan. 


\section{About the Centers}

\author{
The Myrna Brind Center of Integrative Medicine \\ at Jefferson Health \\ Thomas Jefferson University \\ 925 Chestnut Street \\ Suite 120 \\ Philadelphia, PA 19107 \\ Phone: 215-955-2221
}

Website: http://hospitals.jefferson.edu/departments-andservices/myrna-brind-center-of-integrative-medicine/

\section{Brind-Marcus Center of Integrative Medicine at Jefferson Health \\ 789 E. Lancaster Avenue \\ Villanova, PA 19085 \\ Phone: 1-800-JEFF-NOW \\ Website: www.jefferson.edu/hospitals/departments-and- services/brind-marcus-center.html \\ For more information, contact: \\ Daniel A. Monti, MD, MBA \\ Senior Vice President and The Ellen and Ron Caplan \\ Director \& Professor \\ Thomas Jefferson University and Jefferson Health \\ Myrna Brind Center of Integrative Medicine, \\ Philadelphia, PA \\ Brind-Marcus Center of Integrative Medicine, \\ Villanova, PA \\ Phone: 215-955-4410}

MRI scanner are virtually endless. Every integrative approach and every disorder we treat can be evaluated using the PET-MRI scanner. It will help us to better understand the underlying pathophysiology of diseases and show how integrative medicine approaches can help reduce or eliminate the effects of these diseases and improve the physical and mental health of our patients."

\section{Collaboration Key to Success}

Collaboration has been critical for the success of the Myrna Brind Center of Integrative Medicine and the establishment of the new Brind-Marcus Center of Integrative Medicine. Dr. Monti stated: "It has been so important to collaborate whenever there is an opportunity to partner with other departments and institutions and to grow and learn as we go. This spirit of collaboration, I believe, is one of the factors that the Marcus Foundation found appealing about our approach. We have built a relationship with the Marcus Foundation over the past several years, and our current endeavor builds upon previous successes we have had with them, as well as other external funders." He added, "Bernie Marcus is a great visionary who has served the local, national and even international community."

Initially, the Myrna Brind Center of Integrative Medicine partnered with the Marcus Foundation on a phase 1 trial exploring the use of high-dose intravenous vitamin $\mathrm{C}$ with patients who had pancreatic cancer. Collaboration then continued around a discussion about advancing the integrative medicine model and the potential value of a second integrative medicine site. "We are starting to see the fruits of our labor across several initiatives, and having the Marcus Foundation as a partner is tremendous," Dr. Monti said.

Other important elements for success in building and expanding the integrative medicine model have included: a pioneering spirit; testing therapies to prove efficacy; and initiating novel endeavors, such as exploring the use of micronutrients as supportive therapies for patients. "Novel endeavors such as these have raised awareness of the kind of work that we are doing here and sends a message to both patients and clinical partners that we are willing to explore whatever is necessary to help patients who are in difficult positions," Dr. Monti said.

\section{Conclusion}

In the spirit of collaboration, Dr. Monti stated that the structure of integrative medicine provided at the two Centers is serving as a national model to help other clinicians and institutions foster their own integrative medicine programming. Dr. Monti commented:

I, along with other clinical leaders here at Jefferson, am constantly involved with national meetings and symposia where we encounter people from both academic health and private centers who are trying to figure out what steps to take next and how to expand their integrative medicine models. We also publish in both the academic and lay press so that we can share what we are learning with other people in the field. People are eager to come and see what we are doing here and want to understand how to take their centers to the next level. We like being able to share that with people and will continue to serve as a model.

Dr. Monti concluded: "Our goal is to extend the bounds of good medicine, and I think that we are continually achieving that here at our Centers. Our biggest job now is to avoid becoming complacent with these successes and to keep pushing the envelope for the next series of advances."

Jane Hart, MD, is a clinical instructor in internal medicine at Case Western Reserve University School of Medicine, in Cleveland, Ohio. 